\title{
Modelling of anti-tumour immune response: Immunocorrective effect of weak centimetre electromagnetic waves
}

\author{
O.G. Isaeva* and V.A. Osipov \\ Bogoliubov Laboratory of Theoretical Physics, Joint Institute for Nuclear Research, Dubna, \\ Moscow Region, Russia
}

(Received 27 July 2007; final version received 28 July 2008)

\begin{abstract}
We formulate the dynamical model for the anti-tumour immune response based on intercellular cytokine-mediated interactions with the interleukin-2 (IL-2) taken into account. The analysis shows that the expression level of tumour antigens on antigen presenting cells has a distinct influence on the tumour dynamics. At low antigen presentation, a progressive tumour growth takes place to the highest possible value. At high antigen presentation, there is a decrease in tumour size to some value when the dynamical equilibrium between the tumour and the immune system is reached. In the case of the medium antigen presentation, both these regimes can be realized depending on the initial tumour size and the condition of the immune system. A pronounced immunomodulating effect (the suppression of tumour growth and the normalization of IL-2 concentration) is established by considering the influence of low-intensity electromagnetic microwaves as a parametric perturbation of the dynamical system. This finding is in qualitative agreement with the recent experimental results on immunocorrective effects of centimetre electromagnetic waves in tumour-bearing mice.
\end{abstract}

Keywords: carcinogenesis; interleukin-2; modelling; anti-tumour immunity; electromagnetic waves

\section{Introduction}

A theoretical investigation of cancer growth under immunological activity has a long history (see, e.g. [1] and the references therein). Most of the known models consider dynamics of two main populations: effector cells and tumour cells [27,44]. Some models include the dynamics of certain cytokines $[3,10,24]$. An important issue of these studies is a variation of the concentration of cytokines during the disease. As is known, tumour growth results in imbalance between the production and the regulation of cytokines as well as in the reduction of the corresponding receptors thus leading to the suppression of the immunological activity. Therefore, the methods for enhancement of both the anti-tumour resistance and the general condition of the immune system are of current clinical and theoretical interest. One of them refers to the use of cytokines, in particular interleukin-2 (IL-2) $[16,18,21,22]$. IL-2 is considered as the main cytokine responsible for the proliferation of cells containing IL-2 receptors and their following differentiation [48]. IL-2 is mainly produced by activated $\mathrm{CD} 4^{+} \mathrm{T}$ cells. There are many evidences that IL-2 plays an important role in specific immunological reactions to alien agents including tumour cells $[28,38,48]$. Clinical trials also show positive treatment effects at low doses

*Corresponding author. Email: issaeva@theor.jinr.ru 
of IL-2 [18,40-42]. At the same time, at high doses treatment with IL-2 may cause serious haematologic violations revealed by anaemia, granulocytopenia, thrombocytopenia, and lymphocytosis.

The first detailed model of the anti-tumour immune response with IL-2 taken into account was proposed by De Boer et al. [10]. It contains 11 ordinary differential equations and 5 algebraic equations and was used to study the role of macrophage-T lymphocyte interactions that are involved in the cellular immune response. The analysis shows a possibility for both tumour regression and uncontrolled tumour growth depending on 'the degree of antigenicity' (the initial size of the T lymphocyte precursor populations that can be stimulated upon introduction of specific antigen).

Afterward, Kirschner and Panetta [24] proposed a simpler model where only three main populations were considered: the effector cells, the tumour cells and IL-2. The model allows them to study effects of immunotherapy based on the use of cytokines together with adoptive cellular immunotherapy (ACI). ACI refers to the injection of cultured immune cells that have anti-tumour reactivity into tumour bearing host [24]. It was found that without immunotherapy the immune system is unable to clear the tumour with low antigenicity (a measure of how different the tumour is from 'self'), while for highly antigenic tumours reduction to a small dormant tumour takes place. When tumour exhibits average antigenicity, stable limit cycles were observed. This implies that the tumour and the immune system undergo oscillations.

Further, in the framework of the model by Kirschner and Panetta [24], Arciero et al. [3] considered a novel treatment strategy known as small interfering RNA (siRNA) therapy. The model [3] consists of a system of non-linear, ordinary differential equations describing tumour cells, immune effectors, the immuno-stimulatory and suppressive cytokines IL-2 and TGF- $\beta$ as well as siRNA. TGF- $\beta$ suppresses the immune system by inhibiting the activation of effector cells and reducing tumour antigen receptors. It also stimulates tumour growth by promoting angiogenesis. siRNA treatment suppresses TGF- $\beta$ production by targeting the mRNA that codes for TGF- $\beta$, thereby reducing the presence and effect of TGF- $\beta$ in tumour cells. The model predicts conditions under which siRNA treatment can be successful in returning TGF- $\beta$ producing tumours to its passive, non-immune evading state.

Recently, a recovery of IL-2 production after the exposure of tumour-bearing mice to low-intensity centimetre waves was experimentally observed [17]. This indicates that exposure to centimetre electromagnetic waves may be used for an enhancement of the antitumour immune response. In experiments, solid tumours were formed by means of hypodermic transplantation of the ascitic Ehrlich's carcinoma cells. Notice that previous investigations of effects of low-intensive microwave radiation also show the immunomodulating effects at certain frequency ranges and intensities (see, e.g. $[9,26])$. These findings stimulate our interest to study the influence of weak centimetre electromagnetic waves on tumour-immune dynamics. Actually, the influence of electromagnetic radiation (EMR) depends on the type of radiation, a distance from the radiation source (far-field vs. near-field exposure conditions), frequency range, sizes and shapes of objects. Evidently, it is a hard problem to take properly into account all these factors within any theoretical description. In this paper, we offer a reasonable phenomenological approach.

First of all, we formulate an appropriate mathematical model of anti-tumour immune response with the IL-2 taken into account (Section 2). To this end, we follow the scheme of intercellular cytokine-mediated interaction in cellular immune response proposed by Wagner et al. [48] which was modified by taking into account co-stimulatory factors such as B7/CD28 and CD40/CD40L instead of IL-1 (see, e.g. [28,38]). The analysis of the model is presented in Section 3. In Section 4, we discuss a possibility of immunomodulating effect 
of weak radiofrequency electromagnetic radiation (RF EMR) considering the influence of irradiation as a parametric perturbation of the initial dynamical system.

\section{Model}

We describe the dynamics of cellular populations participating in formation of cytotoxic effector cells and cytokines mediating these reactions in accordance with a scheme presented in Figure 1. Some important remarks should be done. Generally, the population of T cells is divided into two subpopulations: helper T cells (HTL) that express marker CD4 on their surface and cytotoxic T cells (CTL) that express CD8 marker [38]. CTL specifically recognize complexes of antigen (AG) with major histocompatibility complex (MHC, in human being - HLA human lymphocyte antigens) class I on the surface of alien or tumour cell and destroy them through this interaction. In contrast to CTL, HTL recognize complexes AG-MHC II on tumour cell and play a regulatory role in the expansion of CTL.

In order to stimulate both HTL and CTL against tumour antigen, it must be presented via MHC classes I and II molecules expressed by professional antigen-presenting cell (APC). There are three main types of professional APCs: dendritic cells, macrophages and B cells. Dendritic cells and, to a lesser extent, macrophages have the broadest range of antigen presentation and are probably the most important APC. They exist as immature (iAPC) and mature (mAPC) forms.

The dynamical equations for immature $\operatorname{APC}(m)$ and mature $\operatorname{APC}(M)$ are written as

$$
\begin{gathered}
\dot{m}=V_{m}-\beta_{m} m-\gamma_{m} m T, \\
\dot{M}=\gamma_{m} m T-\beta_{M} M .
\end{gathered}
$$

In (1), $V_{m}$ characterizes a steady inflow of iAPC from monocytes which in turn are formed from stem cells in the bone marrow. The second term describes iAPC death rate. iAPC phagocytose AG, degrade it, and present their fragments at the plasma membrane using MHC molecules upon maturation. Simultaneously, they express co-stimulatory

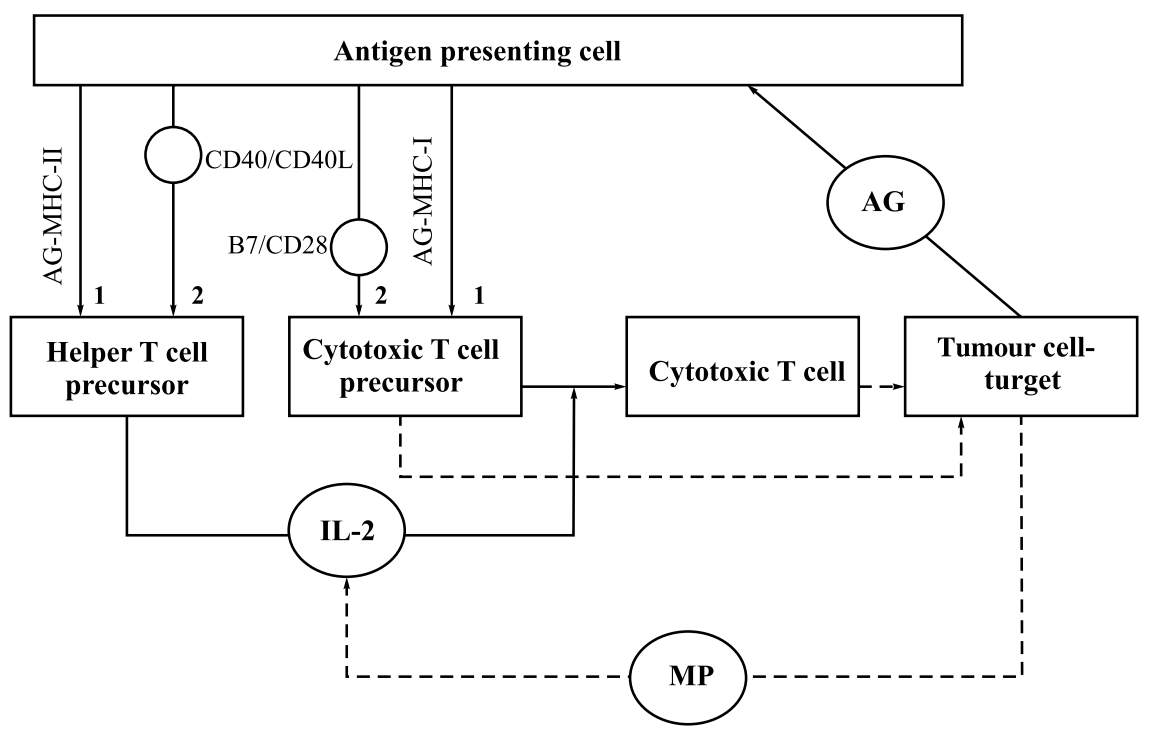

Figure 1. A scheme of the T-cell mediated immune response. 
molecules such as B7 and CD40 [28,38]. Thus iAPC become mAPC expressing both complexes AG-MHC-I and -II as well as co-stimulatory molecules which are recognized by specific receptors on T cells. The rate of transfer from iAPC to mAPC is described by the third term in (1) where $T$ is the number of tumour cells. The concentration of antigen is supposed to be proportional to the number of tumour cells. The production rate of mAPC in (2) is equal to the rate of transfer. The mAPC death rate is described by the second term.

Dynamics of HTL precursors $(H)$ and IL-2 $\left(I_{2}\right)$ is chosen to be

$$
\begin{gathered}
\dot{H}=V_{H}-\beta_{H} H \\
\dot{I}_{2}=\gamma_{H} H M-\tilde{\alpha}_{L} L I_{2}-\gamma_{T} T I_{2} .
\end{gathered}
$$

In (3), $V_{H}$ characterizes the inflow of HTL precursors (HTLP) from stem cells. The second term shows the death rate of HTLP. As a result of interaction between complex AG-MHC-II on mAPC and HTLP (see signal 1 in Figure 1) in the presence of a number of co-stimulatory molecules CD40 (signal 2), activated HTLP produce lymphokines (including IL-2) and corresponding receptors. A similar production is observed when mAPC presents antigen with MHC-I molecule to cytotoxic T cells precursor (CTLP) in the presence of co-stimulatory molecules B7 binding to CD28 markers. The interaction between IL-2 and corresponding receptors on activated T lymphocyte precursors (HTLP and CTLP) induces their proliferation and differentiation into mature T lymphocytes (HTL and CTL). In order to simplify consideration, we omit an equation for HTL activated by tumour antigen assuming that the proliferation of HTL in response solely to IL-2 is absent. This is based on the fact that the levels of expression of IL-2 receptors on HTL are substantially lower than those observed on CTL [30]. Hence, the new AG stimulation is required to support the proliferation of HTL. In addition, our analysis shows that the exclusion of the equation for activated HTL does not influence the tumour growth dynamics within the model. Thus, HTLP stimulated by MAPC are assumed to perform the role of IL-2 producers. We suggest that the concentration of IL-2 grows linearly with HTLP and mAPC [first term in (4)]. As long as IL-2 is a short-distance cytokine, it is supposed that target cells CTL $(L)$ effectively consume IL-2. For this reason, we neglect the term presenting loss rate of IL-2. We also consider in (4) the diminution of IL-2 molecules (third term) as a result of interaction with prostaglandins, immuno-suppressing substances which both suppress the production of IL-2 and directly destroy its molecules [37]. Notice that concentration of prostaglandins is supposed to be proportional to the number of tumour cells $T$.

Let us formulate the dynamical equation for CTL $(L)$. Similarly to the Refs. $[7,13,27,31,32,34]$, we suggest that CTL-tumour cell interaction follows enzymatic kinetics, that is

$$
L+T \underset{\gamma-L}{\stackrel{\gamma_{L}}{\rightleftarrows}} L T \stackrel{\tilde{\gamma}_{L}}{\longrightarrow} P+L
$$

Indeed, CTL can be bound to tumour cell either reversibly (forward and backward reactions with the corresponding rates $\gamma_{L} L T$ and $\gamma_{-L}(L T)$, tumour cells are not 'suffering') or irreversibly ( $L T$ complex is formed) inducing cell death [2,38]. CTL kill tumour cells via one of two main mechanisms. The first one is based on the secretion of perforines. Perforines are embedded into the membrane of tumour cells and form pores thus clearing a way for penetrating water. $L T$ complex dissociates into 'doomed' tumour cell $(P)$ and CTL $(L)$ with a rate $\tilde{\gamma}_{L}(L T)$. Tumour cell swells and gets killed while CTL looks for the new target. The second mechanism involves programmed cell death (apoptosis) through the Fas/Fas ligand pathway. Thus, we introduce an additional equation for 'substratum-enzyme 
complex' $(L T)$ and the equation for immune cells reads

$$
\begin{gathered}
\dot{L}=V_{L}-\beta_{L} L+\alpha_{L} L I_{2}-\gamma_{L} L T+\gamma_{-L}(L T)+\tilde{\gamma}_{L}(L T), \\
(\dot{L} T)=\gamma_{L} L T-\gamma_{-L}(L T)-\tilde{\gamma}_{L}(L T) .
\end{gathered}
$$

In (5), $V_{L}$ characterizes the constant inflow of CTL into the tissue. The second term describes the death rate of CTL. The population of CTL increases due to its proliferation in the presence of IL-2 [third term in (5)]. The remaining terms in (5) describe the CTL-tumour cell interactions. As is seen, Equation (5) describes an expansion of CTL in the presence of IL-2 without antigen stimulation. A similar consideration was presented within the programmed proliferation model by Wodarz and Thomsen [50]. They suggested that the interaction with infected cell transfer CTLP to population of proliferating CTL, which undergo a limited number of divisions without AG stimulation before the differentiation into effector cells. Thus, they use separate equations for population of CTLP, effector cells and $n$ intermediate populations of CTL that passed $i=1,2, \ldots, n$ divisions.

Finally, the population of tumour cells is described by

$$
\dot{T}=-\alpha_{T} T \ln \frac{\beta_{T} T}{\alpha_{T}}-\gamma_{L} T L+\gamma_{-L}(L T) .
$$

Notice that some studies include exponential law to describe tumour growth (see, e.g. $[13,43,44])$. When tumour cells grow in conditions of an interior competition one has to use the limiting growth laws, for instance logistic or Gompertzian $[24,27,46]$. In our model, we prefer to choose the Gompertzian law [the first term in (7)]. This allows us to avoid the regime of tumour autoregression under immunological activity only. Such outcome would contradict numerous clinical experiments. As another reason, clinical and experimental observations show that the growth of some tumours is fitted by the Gompertzian function $[19,33]$.

It should be mentioned that we do not consider here processes of angiogenesis (vascular growth), invasion and metastasis, which are of importance at late (III-IV) stages of the tumour growth. Actually, inclusion of processes of vascular growth and invasion requires serious extension of the model to describe dynamics of cytokines, enzymes and other components regulating these processes. Besides, it would be necessary to take into account spatial migration of cell populations during the process of invasion (see, e.g. [8]). Therefore, the system of Equations (1)-(7) is valid for the description of early stages of the tumour growth when the processes of angiogenesis, invasion and metastasis are not of critical importance. This model allows us to study the different regimes of early immunological activity. However, the formulated model consists of seven differential equations and a great number of model parameters. This makes it difficult to analyse even qualitatively. Therefore, trying to decrease the number of the model equations we will make some simplifying assumptions.

First of all, for lingering diseases one can consider $(L T)(t)$ as rapid variable. In other words, it rapidly reaches its stationary value which remains fixed during the time of the immune response. In this case, $(\dot{L} T)=0$ and one obtains from (6) that $(\overline{L T})=\gamma_{L} L T /\left(\tilde{\gamma}_{L}+\gamma_{-L}\right)$. Substituting this expression in (5) and (7) one finally gets

$$
\begin{gathered}
\dot{L}=V_{L}+\alpha_{L} L I_{2}-\beta_{L} L, \\
\dot{T}=-\alpha_{T} T \ln \frac{\beta_{T} T}{\alpha_{T}}-\gamma_{L}^{\prime} L T,
\end{gathered}
$$

where $\gamma_{L}^{\prime}=\gamma_{L} \tilde{\gamma}_{L} /\left(\gamma_{-L}+\tilde{\gamma}_{L}\right)$. 
Let us assume that $m(t), M(t)$ and $H(t)$ are also in quasi steady states. In this case, Equation (4) is written as

$$
\dot{I}_{2}=\frac{\alpha_{I_{2}} T}{T+K_{T}}-\tilde{\alpha}_{L} L I_{2}-\gamma_{T} T I_{2}
$$

where expressions for $\alpha_{I_{2}}=\gamma_{H} V_{H} V_{m} /\left(\beta_{H} \beta_{M}\right)$ and $K_{T}=\beta_{m} / \gamma_{m}$ follow from the equations $\dot{m}=0, \dot{M}=0$ and $\dot{H}=0$.

Finally, the model becomes much simpler and contains only three main Equations (8)-(10). Nevertheless, it incorporates the most important modern concepts of tumour-immune dynamics including the influence of IL-2 dynamics. The first two equations resemble the famous predator-prey model with tumour cells as 'victims'. As is seen, the growth rate of 'predators' (CTL population) depends on the concentration of IL-2. In (8), we take into account the steady influx of CTL likewise some other considerations (see, e.g. [27,44]). Let us mention once more that, at first glance, such description ignores the preliminary antigen stimulation. In fact, this stimulation is considered in (10) through the first term where IL-2 production depends on tumour size. We use the hyperbola that allows us to take into account a limitation in stimulation of the immune system by the growing tumour. At small $T$ the growth rate is linear in tumour size while for big tumour $\left(T \gg K_{T}\right)$ it tends to be a constant value. The last term in (10) reflects a destruction of IL-2 by metabolic products of tumour cell which are proportional to the concentration of tumour cells [37].

\subsection{Parameter set}

An important question is the choice of parameters. The dynamics of disease is very sensitive to parameters in Equations (8)-(10). The used values are given in Table 1. Some values were estimated by using the available experimental data. In particular, the growth parameters of ascitic Ehrlich carcinoma $\alpha_{T}$ and $\beta_{T}$ were obtained from the experimental data found in Lobo's results where the Ehrlich ascites tumour cell line was cultured in vitro [29]. Using the least-squares method, we fitted the experimental data by Gompertzian curve. The death rate of CTL was estimated using the relation $\beta_{L}=1 / \tau$ where $\tau$ is their known average lifetime. The rate of steady inflow of CTL was calculated from the relation $V_{L}=\beta_{L} L_{\text {free }}$ where $L_{\text {free }}$ (the number of CTL capable to recognize carcinoma specific antigen in the organism without tumour) was estimated to be about $2.4 \times 10^{5}$ cells using the data for the number of $\mathrm{CD} 8^{+} \mathrm{T}$ cells in spleen of mice [5] and a percent value of $\mathrm{T}$ cells specific for tumour type [14]. For the rest of parameters we chose values most appropriate to our model. Current medical literature and sensitivity analysis (see Subsection 3.3) allow us to conclude that the corresponding interactions are of importance in the description of immune response.

\section{Non-dimensionalization, steady state and sensitivity analysis}

\subsection{Scaling}

For convenience, let us introduce dimensionless variables and parameters as follows: $T^{\prime}=T / T_{0}, L^{\prime}=L / L_{0}, I_{2}^{\prime}=I_{2} / I_{20}$, and $t^{\prime}=t / \tau$ where $T_{0}=2.6 \times 10^{6}$ cells, $L_{0}=10^{6}$ cells, $I_{20}=2 \times 10^{7}$ cells, and $\tau=\beta_{L}^{-1}$. The time-scale factor $\tau$ is chosen on the basis that the mean lifespan of CTL is about 3 days and the similar time is needed for the proliferation of CTL and IL-2 production $[6,11]$. 


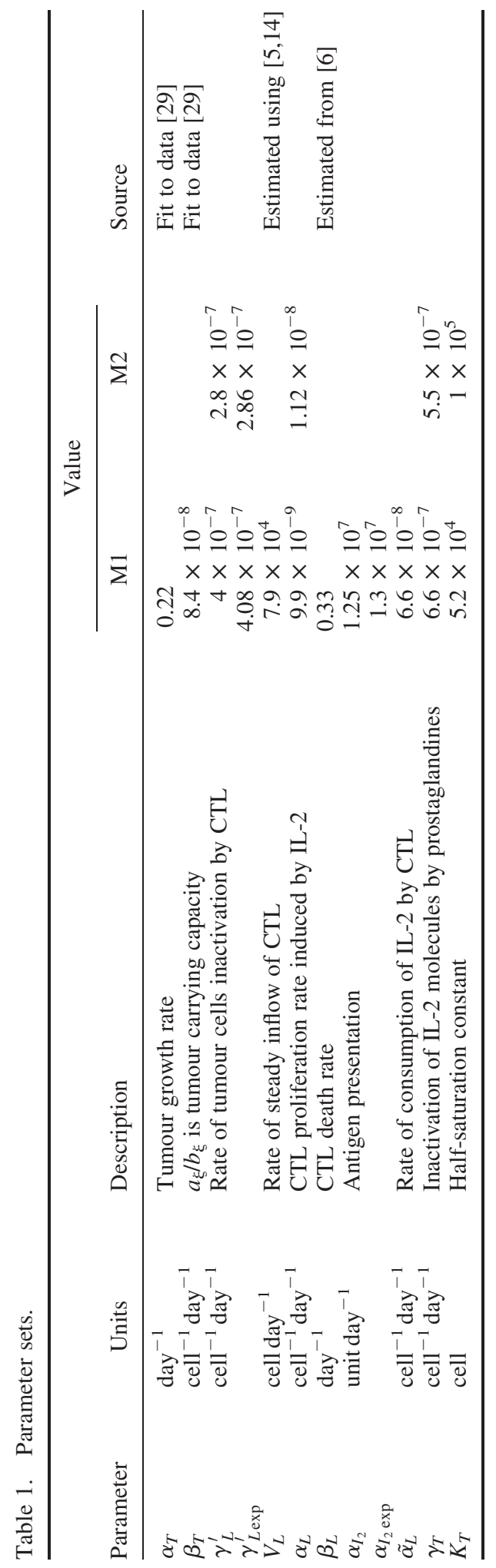


Dropping primes for notational clarity, one finally obtains the following scaled model

$$
\begin{gathered}
\dot{T}=-h_{1} T \ln \frac{h_{2} T}{h_{1}}-h_{3} T L, \\
\dot{L}=h_{4}+h_{5} L I_{2}-L, \\
\dot{I}_{2}=\frac{h_{6} T}{T+h_{9}}-h_{7} L I_{2}-h_{8} T I_{2},
\end{gathered}
$$

where $h_{1}=\alpha_{T} / \beta_{L}, h_{2}=\beta_{T} T_{0} / \beta_{L}, h_{3}=\gamma_{L}^{\prime} L_{0} / \beta_{L}, h_{4}=V_{L} / \beta_{L} L_{0}, h_{5}=\alpha_{L} I_{20} / \beta_{L}, h_{6}=$ $\alpha_{I_{2}} / \beta_{L} I_{20}, h_{7}=\tilde{\alpha}_{L} L_{0} / \beta_{L}, h_{8}=\gamma_{T} T_{0} / \beta_{L}$ and $h_{9}=K_{T} / T_{0}$.

\subsection{Steady states analysis}

Let us perform a steady state analysis of the system of Equations (11)-(13) by using isoclines. We consider the phase plane $T L$ to reflect interactions between two main populations: tumour cells and CTL. In this case, the equations for main isoclines read

$$
\begin{gathered}
\left(h_{4}-L\right)\left(T+h_{9}\right)\left(h_{7} L+h_{8} T\right)+h_{5} h_{6} T L=0, \\
T=0, \quad L=-\frac{h_{1}}{h_{3}} \ln \frac{h_{2} T}{h_{1}} .
\end{gathered}
$$

The fixed points are situated at the intersections of isoclines (14) and (15). Our analysis shows that the systems $(11)-(13)$ have an unstable point $\left(0, h_{4}, 0\right)$ for any choice of parameters. This point lies at the intersection of isoclines (14) and $T=0$.

We consider $\alpha_{I_{2}}$ as a varying parameter to present possible model outcomes. In fact, $\alpha_{I_{2}}$ features the antigen presentation. Indeed, it is proportional to $\gamma_{H}$ which characterizes the probability of interaction between mAPC and HTLP. In turn, this probability depends on the expression of AG-MHC-II complexes on the surface of APC. The antigen presentation by APC is considered as one of important factors in the immune response to tumour. Tumour cells develop a number of mechanisms to escape recognition and elimination by immune system. One of them is the loss or down-regulation of MHC classes I and II molecules presenting AG on tumour cells. This mechanism prevents lymphocytes from recognizing tumour cells [38]. If tumour cells do not possess antigens of MHC-II, an activation of HTL depends on the processing of tumour antigens by APC.

A bifurcation diagram for the dimensionless parameter $h_{6}$ is presented in Figure 2 where the function $h_{6}(T)$ is obtained by substitution of $L$ from (15) into (14). As is seen, there are three bifurcation points. Therefore, one can distinguish four main dynamical regimes. For a low antigen presentation $\left(h_{6}<h_{6 \mathrm{~min}}\right)$, the system of Equations (11)-(13) has two fixed points: a saddle point $\left(0, h_{4}, 0\right)$ and an improper node $\left(T_{3}, L_{3}, I_{23}\right)$. This means that the population of tumour cells is able to escape from the immune response under IL-2 deficiency. The tumour grows and the immune system becomes suppressed. In the region $h_{6 \min }<h_{6}<h_{6 \max }$ corresponding to a medium antigen presentation there appear two additional fixed points: a stable spiral $\left(T_{1}, L_{1}, I_{21}\right)$ and an unstable saddle $\left(T_{2}, L_{2}, I_{22}\right)$. Therefore, different regimes can exist depending on the initial conditions. First, when the initial size of CTL population is sufficiently large the regression of tumour up to a small fixed size takes place (the dynamical equilibrium between tumour and immune system is reached). In this case, the tumour manifests itself via the excited immune system. Second regime appears when initial number of CTL is not large enough 


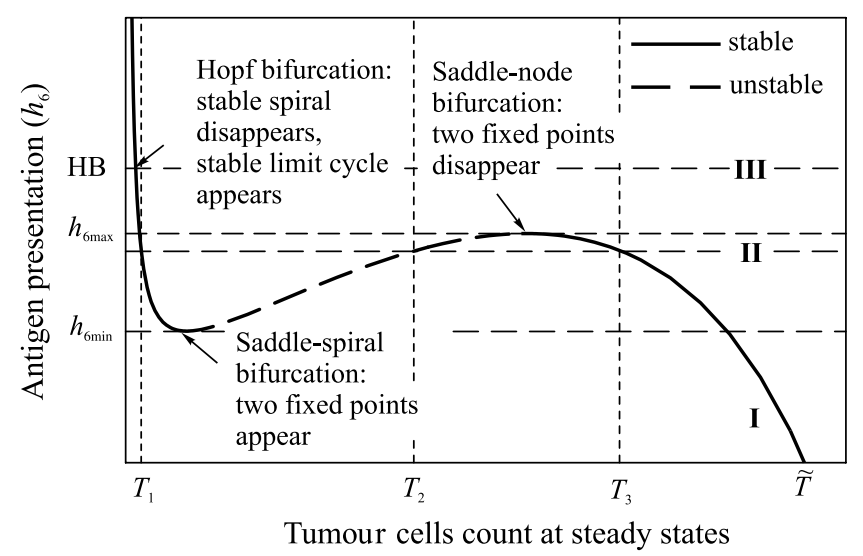

Figure 2. The bifurcation diagram varying the antigen presentation $\left(h_{6}\right)$. For $h_{6}<h_{6 \min }$ there is only one steady state - improper node (region I). When $h_{6 \min }<h_{6}<h_{6 \max }$, there are two stable steady states - improper node and spiral as well as an unstable (saddle) point (region II). For $h_{6}>h_{6 \max }$ only one steady state, the stable spiral remains (region III). For $h_{6}>$ HB the stable spiral passes to the stable limit cycle.

to drive the system at the dynamical equilibrium point $\left(T_{1}, L_{1}, I_{21}\right)$, which is a stable spiral. Thus, the tumour grows to a highest possible size defined by conditions of restricted feeding. The dynamical equilibrium between the tumour and immune system is reached at the fixed point $\left(T_{3}, L_{3}, I_{23}\right)$ that is an improper node. In the case of a high antigen presentation $\left(h_{6}>h_{6 \max }\right)$, the fixed points $\left(T_{2}, L_{2}, I_{22}\right)$ and $\left(T_{3}, L_{3}, I_{23}\right)$ disappear. As a result, there are two fixed points: a saddle point $\left(0, h_{4}, 0\right)$ and a stable spiral $\left(T_{1}, L_{1}, I_{21}\right)$. In this case, a decrease in tumour size is found when the equilibrium between the tumour and the immune system is established (dormant tumour). Finally, let us discuss the case of a high antigen presentation level $\left(h_{6}>\mathrm{HB}\right)$ when Hopf bifurcation occurs and stable spiral $\left(T_{1}, L_{1}, I_{21}\right)$ becomes unstable spiral. Integral curves tend to stable limit cycle and, accordingly, we observe oscillations in small tumour size, number of CTL and the concentration of IL-2. This means that the immune system is able to prevent tumour from uncontrolled growing. This also corresponds to the dormant tumour.

\subsection{Sensitivity analysis}

The sensitivity analysis has been carried out to test which components of the model (8)-(10) contribute most significantly to tumour dynamics. We altered each parameter (taken separately) from its estimated value (Table 1, M1) by $1 \%$ and calculated the change in the tumour size after 30 days. The results are shown in Figure 3. As is seen, the system is most sensitive to the tumour growth rate $\alpha_{T}$ and the CTL death rate $\beta_{L}$.

We found lesser (yet remarkable) sensitivity to the following parameters: the rate of tumour cells inactivation by CTL $\gamma_{L}^{\prime}$, the CTL proliferation rate $\alpha_{L}$, the antigen presentation $\alpha_{I_{2}}$ as well as the rate of inactivation of the IL-2 molecules by prostaglandins $\gamma_{T}$. The system is of little sensitivity to the consumption of IL-2 $\tilde{\alpha}_{L}$ and the half-saturation constant $K_{T}$. What is important for our consideration, the parameters $\gamma_{L}^{\prime}$ and $\alpha_{I_{2}}$ belong to the second group. This means that even a small variation of either the antigen expression on tumour cells or the antigen presentation by APC will markedly affect tumour dynamics. Based on both bifurcation and sensitivity analysis, we will associate the region I in Figure 2 with a weak immune response, and the region II with the strong immune response. 


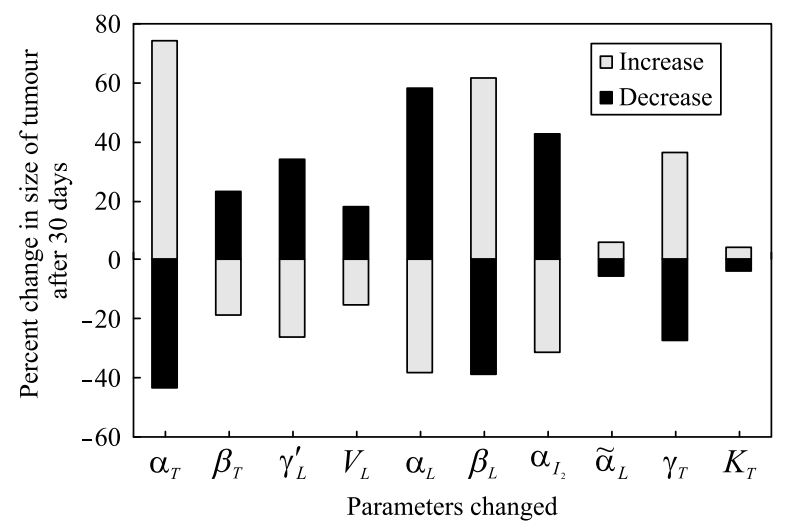

Figure 3. The sensitivity analysis for the parameter set M1 in Table 1. The tumour size is more sensitive to tumour growth rate variable $\alpha_{T}$, to CTL death rate $\beta_{L}$, to inactivation of tumour cells by CTL $\gamma_{L}$, to antigen presentation $\alpha_{I 2}$, to CTL proliferation variable $\alpha_{L}$ as well as to the rate of inactivation of the IL-2 molecules by prostaglandins $\gamma_{T}$.

The region III is associated with the case of dormant tumour when the immune system is able to handle the tumour size.

In conclusion, it is interesting to examine how alterations of either $\alpha_{T}$ or $\gamma_{L}^{\prime}$ affect the model regimes. Let us introduce a variable $\tilde{T}$

$$
\tilde{T}=\frac{h_{1}}{h_{2}} \exp \left(-\frac{h_{3} h_{4}}{h_{1}}\right),
$$

which is a zero of the function $h_{6}(T)$ (see Figure 2). The bifurcation diagram for dimensionless parameters $h_{6}$ versus $h_{1}$ is shown in Figure 4(a). As is seen, both $h_{6 \min }$ and $h_{6 \max }$ increase with $h_{1}$. For small rate of tumour growth, the region II diminishes and $\tilde{T}$ decreases in (16). In this case, the region II becomes inessential and the dynamical behaviour is determined by the regions I and III. The final tumour size in the region I becomes small in comparison with the case of rapidly growing tumour. Besides, in the region III HB increases with $h_{1}$ [see Figure 4(a)]. This means that slowly growing tumours are not able to evade even weak immune supervision. In the case of high rate of tumour growth, the region II markedly extends

(a)

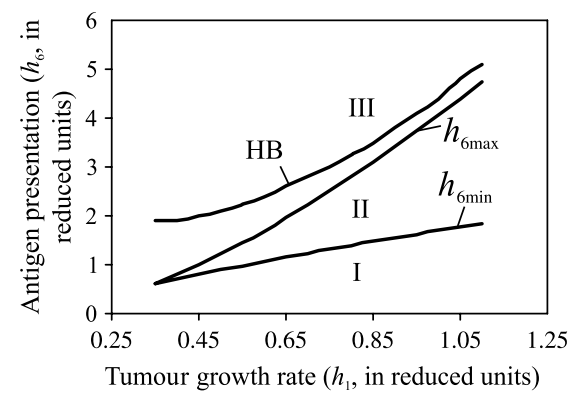

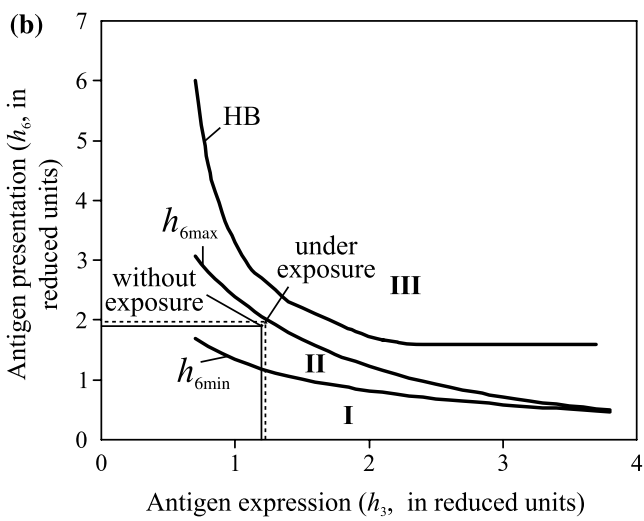

Figure 4. The bifurcation diagram $h_{6}$ versus $h_{1}$ (a). The bifurcation diagram $h_{6}$ versus $h_{3}$ and the variation of steady state regime under exposure to low-intensive RF EMR (b). Region I - weak immune response, region II - strong immune response and region III - dormant tumour. 
and $\tilde{T}$ increases. Therefore, a high antigen presentation is required to reach the region III corresponding to dormant tumour and the possibility of tumour remission decreases with increasing tumour growth rate. In other words, the rate of tumour growth can give warning of malignance.

The next important characteristic determining the outcome of the disease is the expression of AG-MHC-I complexes on the surface of tumour cells. In our consideration, a level of this expression is characterized by the parameter $\gamma_{L}^{\prime}$. Figure 4(b) shows the bifurcation diagram for $h_{6}$ versus $h_{3}$. As is seen, with $h_{3}$ increasing the region II vanishes and $\tilde{T}$ descends in (16). This means that the immune system is able to handle cancer. For small antigen expression, the strength of the immune response depends on the level of antigen presentation $\left(h_{6}\right)$. Therefore, for tumours with poor immunogenicity (low antigen expression) a high antigen presentation on APC can be responsible for the strong immune response.

\section{Immunocorrective effects of radiofrequency electromagnetic waves}

In this section, we discuss a possible way to take into consideration the influence of low-intensity electromagnetic microwaves within our model. Since the main effects have a complex non-linear dependence on frequency, intensity and other characteristics of EMR we suggest using a phenomenological approach. To justify our consideration let us present an overview of some important biological and physical aspects.

Above all, we would like to stress that our consideration is restricted to the frequency range $8-18 \mathrm{GHz}$ and a low incident power $\sim 1 \mu \mathrm{W} / \mathrm{cm}^{2}$ because namely these characteristics of EMR were explored in recent experiments by Glushkova et al. [17]. Two important experimental findings should be mentioned. First, both the concentration of IL-2 in the serum of tumour-bearing mice and the production of this cytokine were found to be normalized after exposure to microwaves. Second, the yield of heat shock proteins72 (HSP-72) by spleencytes was observed in both healthy and tumour-bearing mice exposed to radiation. The last finding is rather surprising and could indicate the presence of cellular stress response under the exposure. As is known, HSP play a role of 'molecular chaperones' binding to and stabilizing partially unfolded proteins, thus providing the cell with protection. However, our estimation of the specific absorption rate by using the empirical model by Durney et al. [12] gives $\sim 0.5 \mathrm{~mW} / \mathrm{kg}$ for mouse. In experiments [17], mice were exposed to microwaves daily during 20 days. The duration of the exposure was $1.5 \mathrm{~h}$. It is easy to estimate that during $1.5 \mathrm{~h}$ only $2.7 \mathrm{~J} / \mathrm{kg}$ of electromagnetic energy is absorbed. Therefore, the intensity level used in Ref. [17] is not sufficient for occurring conformation changes. In this case, the question arises: how to explain the appearance of HSP? Unfortunately, this is an open problem yet. Nevertheless, some existing ideas allow us to suggest the following scenario.

In accordance with a hypothesis of the resonant absorption, the electromagnetic energy in microwave (RF) range is absorbed mainly by aqueous environment. Therefore, the observed HSP production could be caused by free radicals in water (see, e.g. [20]). According to Refs. [4,47], free radicals may be produced from water $\left(\mathrm{H}_{2} \mathrm{O}\right)$ by any process that moves clusters of water relative to each other, for instance, the mechanical vibration

$$
\left(\mathrm{H}_{2} \mathrm{O}\right)_{n}\left(\mathrm{H}_{2} \mathrm{O} \leftarrow \mathrm{H}-\mathrm{OH} \rightarrow \mathrm{OH}_{2}\right)\left(\mathrm{H}_{2} \mathrm{O}\right)_{m} \rightarrow\left(\mathrm{H}_{2} \mathrm{O}\right)_{n}(\mathrm{H}_{2} \mathrm{O}+\underbrace{\mathrm{H} \cdot+\cdot \mathrm{OH}}_{\text {free radicals }}+\mathrm{OH}_{2})\left(\mathrm{H}_{2} \mathrm{O}\right)_{m}
$$


In the case of low-intensive EMR, small mechanical vibrations of water clusters may result from non-radiating transitions of excited molecules. It should be stressed that at low incident power of EMR very low concentrations of free radicals will be formed. This is very important for getting the therapeutic effect because the perturbations in concentrations of free radicals should not exceed physiological levels. In this case, mechanisms of natural antioxidant defence are able to reduce oxidative stress. For example, melatonin is found to mediate the inactivation of free radicals by stimulating some important antioxidative enzymes [36]. Besides, melatonin is able to activate helper T lymphocytes thereby increasing the production of IL-2 and IFN- $\gamma$ [15]. This could explain the experimentally observed recovery of IL-2 production. There is also a different possible mechanism of antioxidant defence when free radicals activate such nucleus transcription factors as NFAT and NFKB (see Ref. [45] and the references therein). Indeed, NFKB and NFAT induce the expression of the antioxidant genes [20,45]. It has been recently observed in experiment that the production of NFKB actually increases as a result of exposure to weak RF EMR [23]. Notice that NFAT and NFkB are transcriptional regulators of the IL-2 gene [25,39]. Therefore, additionally to the antigen stimulation, these factors can be also activated by EMR-induced free radicals thereby enhancing the production of both IL-2 and very likely IFN- $\gamma$.

Let us revert to the model. In order to reflect the influence of EMR, we assume to vary two basic model parameters $\gamma_{L}^{\prime}$ and $\alpha_{I_{2}}$. Let us remind that $\gamma_{L}^{\prime}$ represents the destruction rate of tumour cells by CTL. With growing production of IFN- $\gamma$ the expression of molecules MHC classes I and II on tumour cells increases thus enhancing their recognition by CTL [35]. In addition, HSP-72 also mediate up-regulation of AG-MHC-I complexes on surface of tumour cells [49]. Therefore, the parameter $\gamma_{L}^{\prime}$ should be increased for taking into account the radiation. The parameter $\alpha_{I_{2}}$ characterizes the antigen presentation. Notice that for big tumour sizes $\alpha_{I_{2}}$ determine the rate of the IL-2 production that is enhanced by the melatonin. Therefore, $\alpha_{I_{2}}$ also should be increased. We assume that these parameters remain time-independent and merely increase to the new constant values $\gamma_{L \operatorname{Lxp}}^{\prime}$ and $\alpha_{I_{2} \exp }$. In other words, we suggest that an influence of EMR is effective during all the time between exposures. Unfortunately, it is impossible to extract the values of $\gamma_{L \exp }^{\prime}$ and $\alpha_{I_{2} \exp }$ from existing experiments. Therefore, we will study the role of these parameters by taking into account the fact that the influence of low-intensity EMR is weak. In this case, we use trial values for $\gamma_{L \exp }^{\prime}$ and $\alpha_{I_{2} \exp }$ assuming that $\gamma_{L}^{\prime}$ and $\alpha_{I_{2}}$ are only slightly increased under exposure (by 2 and $4 \%$, respectively, see Table 1). As an additional criterion, the interval of variability of these parameters should be chosen in such a way to prevent the system from passing to the region III where the regime of dormant tumour is realized (see Figure 4(b)).

We present numerical results for two parameter sets M1 and M2 (see Table 1) to illustrate the body specific effects of electromagnetic radiation. Figure 5 shows bifurcation diagrams for both M1 and M2. As is seen, in both cases the system is located in the region of the strong immune response. Hence, the outcome of disease depends on the initial conditions. We assume the same initial numbers of tumour cells and CTL whereas the initial concentration of IL-2 for M1 is taken to be higher than for M2. In this case, the remission of tumour for M1 and progressive growth for M2 are found (see Figures 6 and 7). As is seen from Figure 6, without exposure the dynamical curves for M1 have a character of dumping oscillations. The tumour decreases to a small size corresponding to the stable spiral. Although the tumour growth is handled by the immune system, for the first 20 days the tumour size is high enough (Figure 6(a)). As a result, the IL-2 concentration is smaller than its initial value during this period (Figure 6(c)). At the same time, the population of CTL increases (Figure 6(b)). The results show that tumour cells stimulate immune 


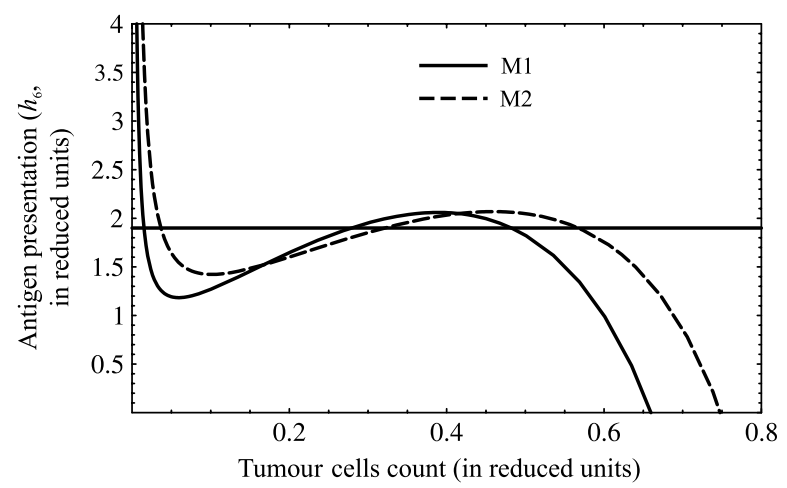

Figure 5. Bifurcation diagrams showing the steady state regimes for the model parameter sets M1 and $\mathrm{M} 2$.

response. This qualitatively agrees with the experimental results [17] where both the decrease of the IL- 2 concentration and the increase of the number of CTL were observed in 20 days of tumour growth.

Figure 6(a) shows that after exposure to weak RF electromagnetic waves during 20 days the tumour size becomes smaller than in the case without exposure. The concentration of IL-2 markedly increases and reaches the initial value on 20th day (Figure 6(c)). Accordingly, the population of CTL also grows up to a larger value in comparison with the case without exposure (Figure 6(b)). Thus, our results show that the concentration of IL-2 is restored as a result of exposure to EMR, which also qualitatively agrees with the experimental observations [17]. It should be mentioned that there are some differences
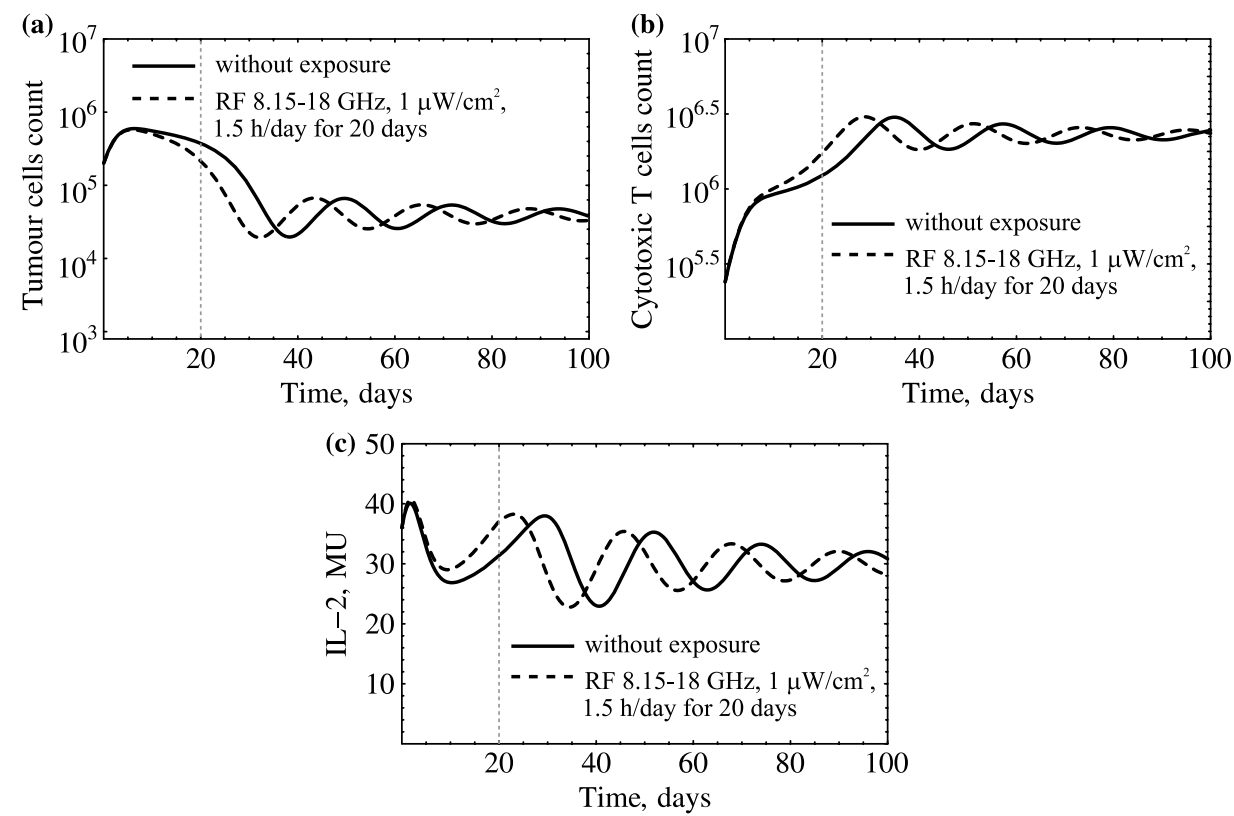

Figure 6. Effects of low-intensive RF EMR: (a) tumour cells, (b) cytotoxic T cells and (c) IL-2 versus time for the parameter set M1. The irradiation occurs during 20 days. Initial conditions: $2 \times 10^{5}$ tumour cells, $2.4 \times 10^{5}$ cytotoxic T lymphocytes, $3.6 \times 10^{7}$ IL-2 units. 

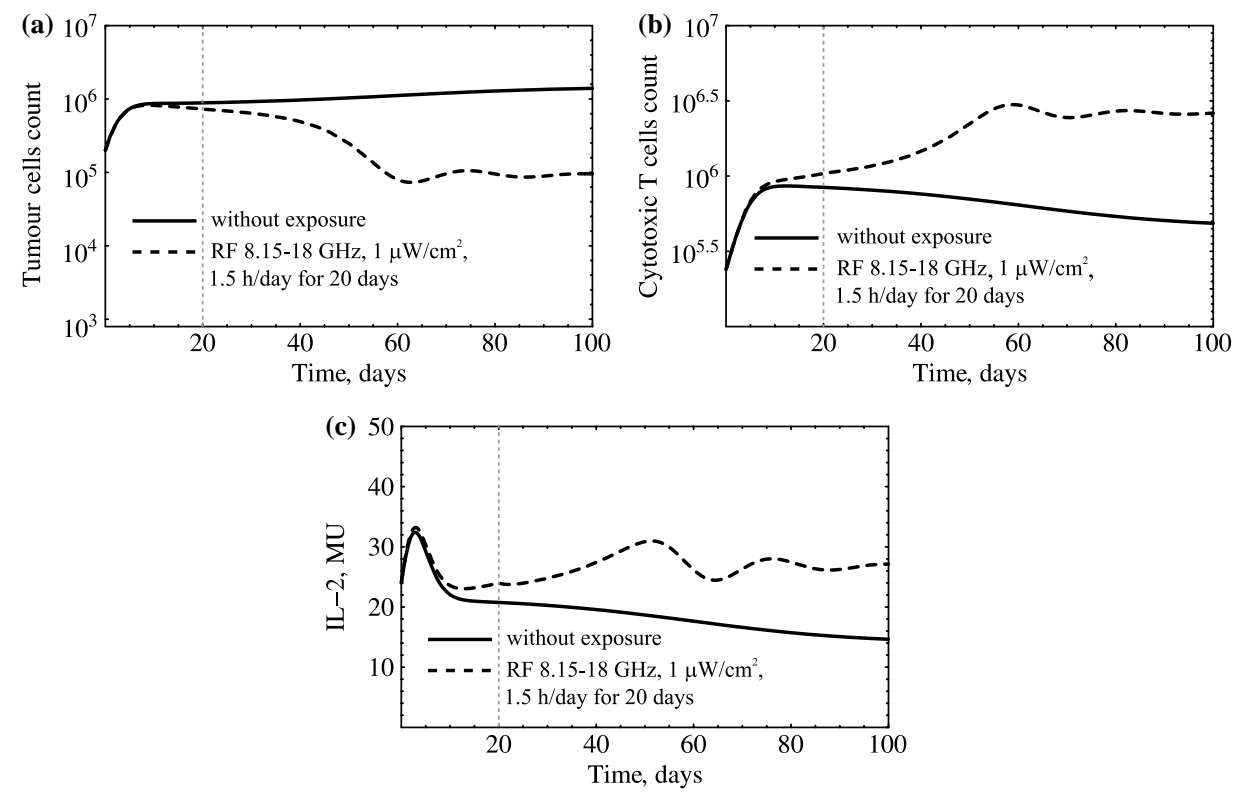

Figure 7. Effects of low-intensive RF EMR: (a) tumour cells, (b) cytotoxic T cells and (c) IL-2 versus time for the parameter set M2. The irradiation occurs during 20 days. Initial conditions: $2 \times 10^{5}$ tumour cells, $2.4 \times 10^{5}$ cytotoxic T lymphocytes, $2.4 \times 10^{7} \mathrm{IL}-2$ units.

between predictions of our model and the experiment. For example, in experiment a decrease of the CTL population in comparison with unexposed mice was found after 20 days of irradiation instead of the increase in our model. It may be that the production of HSP blocking the proliferation is responsible for this observation. The dynamics of HSP is not explicitly taken into account in our model.

In the case of M2, without exposure the tumour grows up to the maximum possible value (Figure 7(a)). At the same time, the population of CTL and the IL-2 concentration decrease (Figure 7(b) and (c)). Nevertheless, initially the tumour stimulates the immune response. Hence, the number of CTL on 20th day of tumour growth is higher than their initial value (Figure 7(b)). As is seen from Figure 7, after cessation of daily exposure to weak RF EMR during 20 days [when the parameters take their normal (initial) values] the dynamical curves tend to the stable spiral, and the tumour remission takes place. At the same time, the population of CTL and the concentration of IL-2 increase in comparison with unexposed cases. Thus, the behaviour of the IL-2 concentration for M2 also qualitatively agrees with experimental observations [17]. It is important that the influence of weak EMR leads to the change of dynamical regime from progressive growth to remission of tumour. This follows from the fact that the number of tumour cells and CTL as well as the IL-2 concentration fall into the basin of attraction of stable spiral after the cessation of exposure. Summarizing, our results show the pronounced immunocorrective effect of the weak RF EMR.

\section{Conclusion}

In this paper, we have formulated the mathematical model for the immune response to the malignant growth with the IL-2 taken into account. It is found that tumour growth rate and the level of antigen expression on tumour cells and APC are important factors determining the dynamics of disease. Four main dynamical regimes are revealed and shown on the 
bifurcation diagram for antigen presentation by APC. For a low antigen presentation, the tumour is able to escape from the immune response. In the case of a medium antigen presentation there exist two regimens of disease depending on both the initial tumour size and the condition of immune system: (1) the regression to small tumour when the dynamical equilibrium is established and (2) a progressive tumour growth to the highest possible size. For a high antigen presentation, the decrease of the tumour size is found when the equilibrium between the tumour and the immune system is established. Additionally, the regime of oscillations in small tumour size, the number of CTL and the concentration of IL-2 are observed due to the presence of stable limit cycle. It is important to note that the regime of full tumour regression as a result of the immune response alone is not admitted within our model. This fact is in agreement with clinical observations where spontaneous regression of tumours is not possible.

In order to illustrate the behaviour of the system with the effects of weak RF EMR taken into account we have chosen two parameter sets so that the system is located in the region II of bifurcation diagram where the result of immune response depends on initial tumour size and the immune system condition. Namely in this region the system is most sensitive to perturbation of the model parameters. We have considered the influence of two model parameters characterizing both the rate of inactivation of tumour cells by CTL and the production of IL-2. Our results show the marked immunocorrective effect of weak RF EMR. In particular, an increase of the IL-2 concentration in comparison with unexposed case and enhancement of the immune response are found. Moreover, it may be expected that the RF EMR at low intensity is low-toxic. Indeed, we found only minor increase of the IL-2 concentration which does not exceed the norm. Nevertheless, the frequency range, intensity and other EMR parameters as well as the regimen of exposure should be carefully estimated to avoid the harmful influence of EMR on the central nervous, cardiovascular and other systems of the body.

\section{Note}

1. Email: osipov@theor.jinr.ru

\section{References}

[1] J.A. Adam and N. Bellomo, A Survey of Models for Tumor-Immune System Dynamics, Birkhäuser, Boston, MA, 1996.

[2] B. Alberts, D. Bray, J. Lewis, M. Raff, K. Roberts, and J.D. Watson, Molecular Biology of the Cell, 3rd edn., New York, Garland Publishing, Inc., 1994, p. 1408.

[3] J.C. Arciero, D.E. Kirschner, and T.L. Jackson, A mathematical model of tumor-immune evasion and siRNA treatment, Disc. Cont. Dyn. Syst.-B 4(1) (2004), pp. 39-58.

[4] H.J. Bakker and H.-K. Nienhuys, Delocalization of protons in liquid water, Science 297 (2002), pp. $587-590$.

[5] A. Casrouge, E. Beaudoing, S. Dalle, C. Pannetier, J. Kanellopoulos, and P. Kourilsky, Size estimate of the ab TCR repertoire of naive mouse splenocytes, J. Immunol. 164 (2000), pp. $5782-5787$.

[6] D.L. Chao, M.P. Davenport, S. Forrest, and A.S. Perelson, A stochastic model of cytotoxic T cell responses, J. Theoret. Biol. 228 (2004), pp. 227-240.

[7] M. Chaplain and A. Matzavinos, Mathematical modeling of spatio-temporal phenomena in tumor immunology, Lect. Notes Math. 1872 (2006), pp. 131-183.

[8] M.A.J. Chaplain, Mathematical models in cancer research, The Cancer Handbook, Chap. 60, Nature Publishing Group, London, 2003, pp. 937-951.

[9] S.F. Cleary, L.M. Liu, and R.E. Merchant, Lymphocyte proliferation induced by radio-frequency electromagnetic radiation under isothermal conditions, Bioelectromagnetics 11 (1990), pp. 47-56. 
[10] R.J. De Boer, P. Hogeweg, F.J. Dullens, R.A. De Weger, and W. Den Otter, Macrophage T lymphocyte interactions in the anti-tumor immune response: A mathematical model, J. Immunol. 134(4) (1985), pp. 2748-2758.

[11] R.J. De Boer, M. Oprera, R. Antia, K. Murali-Krishna, R. Ahmed, and A.S. Perelson, Recruitment times, proliferation, and apoptosis rates during the CD8 $+T$ cell Response to lymphocytic choriomeningitis virus, J. Virol. 75(22) (2001), pp. 10663-10669.

[12] C.H. Durney, M.F. Iskander, H. Massoundi, and C.C. Johnson, An empirical formula for broad-band SAR calculation of prolate spheroidal models of humans and animals, IEEE Trans. Microw. Theory Tech. 27(8) (1979), pp. 758-763.

[13] R. Garay and R. Lefever, A kinetic approach to the immunology of cancer: Stationary states properties of effector-target cell reactions, J. Theoret. Biol. 73 (1978), pp. 417-438.

[14] S. Garbelli, S. Mantovani, B. Palermo, and C. Giachino, Melanocyte-specific, cytotoxic T cell responses in vitiligo: The effective variant of melanoma immunity, Pigment Cell Res. 18 (2005), pp. 234-242.

[15] S. Garcia-Maurino, M.G. Gonzalez-Haba, J.R. Calvo, M. Rafii-El-Idrissi, V. SanchezMargalet, R. Goberna, and J.M. Guerrero, Melatonin enhances IL-2, IL-6, and IFN-gamma production by human circulating CD4 + cells: A possible nuclear receptor-mediated mechanism involving T helper type 1 lymphocytes and monocytes, J. Immunol. 159(2) (1997), pp. $574-581$.

[16] B.L. Gause, M. Sznol, W.C. Kopp, J.E. Janik, J.W. Smith, II, R.G. Steis, W.J. Urba, W. Sharfman, R.G. Fenton, S.P. Creekmore, J. Holmlund, K.C. Conlon, L.A. VanderMolen, and D.L. Longo, Phase I study of subcutaneously administered interleukine-2 in combination with interferon alfa$2 a$ in patients with advanced cancer, J. Clin. Oncol. 14(8) (1996), pp. 2234-2241.

[17] O.V. Glushkova, E.G. Novoselova, O.A. Sinotova, and E.E. Fesenko, Immunocorrecting effect of super-high frequency electromagnetic radiation in carcinogenesis in mice, Biophysics. 48(2) (2003), pp. 264-271.

[18] I. Hara, H. Hotta, N. Sato, H. Eto, S. Arakava, and S. Kamidono, Rejection of mouse renal cell carcinoma elicited by local secretion of interleukin-2, J. Cancer Res. 87 (1996), pp. 724-729.

[19] S. Heegaard, M. Spang-Thomsen, and J.U. Prause, Establishment and characterization of human uveal malignant melanoma xenografts in nude mice, Melanoma Res. 13(3) (2003), pp. 247-251.

[20] M.J. Jackson, A. McArdle, and F. McArdle, Antioxidant micronutrients and gene expression, Proc. Nutr. Soc. 57 (1998), pp. 301-305.

[21] R. Kaempfer, L. Gerez, H. Farbstein, L. Madar, O. Hirschman, R. Nussinovich, and A. Shapiro, Prediction of response to treatment in superficial bladder carcinoma through pattern of interleukin-2 gene expression, J. Clin. Oncol. 14(6) (1996), pp. 1778-1786.

[22] U. Keiholz, C. Scheibenbogen, E. Stoelben, H.D. Saeger, and W. Hunstein, Immunotherapy of metastatic melanoma with interferon-alpha and interleukin-2: Pattern of progression in responders and patients with stable disease with or without resection of residual lesions, Eur. J. Cancer. 30A(7) (1994), pp. 955-958.

[23] M.O. Khrenov, D.A. Cherenkov, O.V. Glushkova, T.V. Novoselova, S.M. Lunin, S.B. Parfeniuk, E.A. Lysenko, E.G. Novoselova, and E.E. Fesenko, The role of transcription factors in the response of mouse lymphocytes to low-level electromagnetic and laser radiations, Biofizika. 52(5) (2007), pp. 888-892.

[24] D. Kirschner and J.C. Panetta, Modeling immunotherapy of the tumor-immune interaction, J. Math. Biol. 37 (1998), pp. 235-252.

[25] R. Konig and W. Zhou, Signal transduction in T helper cells: CD4 coreceptors exert complex regulatory effects on T cell activation and function, Curr. Issues Mol. Biol. 6(1) (2004), pp. 1-15.

[26] N.N. Kositsky, A.I. Nizhelska, and G.V. Ponezha, Influence of high-frequency electromagnetic radiation at non-thermal intensities on the human body (a review of work by Russian and Ukrainian researchers), No place to hide, 3(1) (2001), Supplement www.emfacts.com/ussr_review.pdf

[27] V.A. Kuznetsov, I.A. Makalkin, M.A. Taylor, and A.S. Perelson, Nonlinear dynamics of immunogenic tumors: Parameter estimation and global bifurcation analysis, Bull. Math. Biol. 56(2) (1994), pp. 295-321.

[28] Y. Liu, Y. Ng, and K.O. Lillehei, Cell mediated immunotherapy: A new approach to the treatment of malignant glioma, Cancer Control. 10(2) (2003), pp. 138-147. 
[29] C. Lobo, M.A. Ruiz-Bellido, J.C. Aledo, J. Marquez, I. Nunez de Castro, and F.J. Alonso, Inhibition of glutaminase expression by antisense $m R N A$ decreases growth and tumourigenicity of tumour cells, Biochem. J. 348 (2000), pp. 257-261.

[30] J. Lu, R.L. Giuntoli, II, R. Omiya, H. Kobayashi, R. Kennedy, and E. Celis, Interleukin 15 promotes antigen-independent in vitro expansion and long-term survival of antitumor cytotoxic T lymphocytes, Clin. Cancer Res. 8 (2002), pp. 3877-3884.

[31] A. Matzavinos, M.A.J. Chaplain, and V.A. Kuznetsov, Mathematical modeling of the spatiotemporal response of cytotoxic T-lymphocytes to a solid tumour, Math. Med. Biol. 21 (2004), pp. $1-34$.

[32] S.J. Merril, Foundations of the use of an enzyme-kinetic analogy in cell-mediated cytotoxicity, Math. Biosci. 62 (1982), pp. 219-235.

[33] L. Norton, A Gompertzian model of human breast growth, Cancer. Res. 48 (1988), pp. $7067-7071$.

[34] A. Ochab-Marcinek and E. Gudowska-Nowak, Population growth and control in stochastic models of cancer development, Phys. A. 343 (2004), pp. 557-572.

[35] L. Raffaghello, I. Prigione, P. Bocca, F. Morandi, M. Camoriano, C. Gambini, X. Wang, S. Ferrone and V. Pistoia, Multiple defects of the antigen-processing machinery components in human neuroblastoma: Immunotherapeutic implications, Oncogene. 24 (2005), pp. 4634-4644.

[36] R.J. Reiter, R.C. Carneiro, and C.S. Oh, Melatonin in relation to cellular antioxidative defense mechanisms, Horm. Metab. Res. 29(8) (1997), pp. 363-372.

[37] RESAN Scientific Research Enterprise Scientific research enterprise web site (2003). Available at http://www.anticancer.net/resan/basis.html\#interleukins

[38] I. Roitt, J. Brostoff, and D. Male, Immunology, 6th edn., Mosby, London, 2001, p. 480.

[39] J.W. Rooney, Y.L. Sun, L.H. Glimcher, and T. Hoey, Novel NFAT sites that mediate activation of the interleukin-2 promoter in response to T-cell receptor stimulation, Mol. Cell. Biol. 15 (1995), pp. 6299-6310.

[40] S.A. Rosenberg and M.T. Lotze, Cancer immunotherapy using interleukin-2 and interleukin-2activated lymphocytes, Ann. Rev. Immunol. 4 (1986), pp. 681-709.

[41] S.A. Rosenberg, J.C. Yang, S.L. Toplian, D.J. Schwartzentruber, J.S. Weber, D.R. Parkinson, C.A. Seipp, J.H. Einhorn, and D.E. White, Treatment of 283 consecutive patients with metastatic melanoma or renal cell cancer using high-dose bolus interleukin 2, JAMA. 271(12) (1994), pp. 907-913.

[42] D.J. Schwartzentruber, In vitro predictors of clinical response in patients receiving interleukin2-based immunotherapy, Curr. Opin. Oncol. 5 (1993), pp. 1055-1058.

[43] J. Sherrat and M. Nowak, Oncogenes, anti-oncogenes and the immune response to cancer: A mathematical model, Proc. R. Soc. Lond. B 248 (1992), pp. 261-271.

[44] N. Stepanova, Course of the immune reaction during the development of a malignant tumor, Biophysics. 24 (1980), pp. 917-923.

[45] M. Valko, D. Leibfritz, J. Moncola, M.T.D. Cronin, M. Mazur, and J. Telser, Free radicals and antioxidants in normal physiological functions and human disease, Review is available online at www.sciensdirect.com

[46] H.P. Vladar and J.A. Gonzalez, Dynamic response of cancer under the influence of immunological activity and therapy, J. Theoret. Biol. 227 (2004), pp. 335-348.

[47] V.L. Voeikov, Biological significance of active oxygen-dependent processes in aqueous systems, in Water and the Cell, G.H. Pollack, I.L. Cameron, and D.N. Wheatley, eds., Springer, Dordrecht, 2006, pp. 285-298.

[48] H. Wagner, C. Hardt, K. Heeg, K. Pfizenmaier, W. Solbach, R. Bartlett, H. Stockinger, and M. Rollingoff, T-T cell interactions during CTL responses: $T$ cell derived helper factor (interleukin 2) as a probe to analyze CTL responsiveness and thymic maturation of CTL progenitors, Immunol. Rev. 51 (1980), pp. 215-255.

[49] A.D. Wells, S.K. Rai, S. Salvato, H. Band, and M. Malkovsky, HSP-72-mediated augmentation of MHC class I surface expression and endogenous antigen presentation, Int. Immunol. 10(5) (1998), pp. 609-617.

[50] D. Wodarz and A.R. Thomsen, Does programmed CTL proliferation optimize virus control? Trends Immunol. 26 (2005), pp. 305-310. 


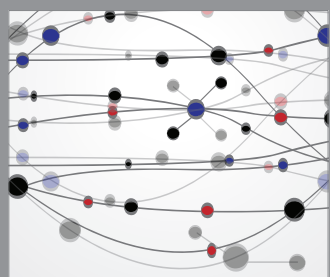

The Scientific World Journal
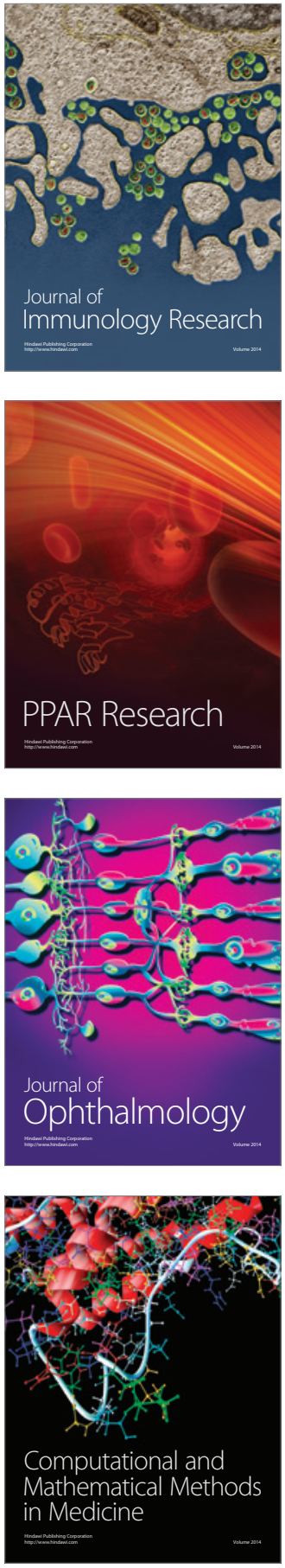

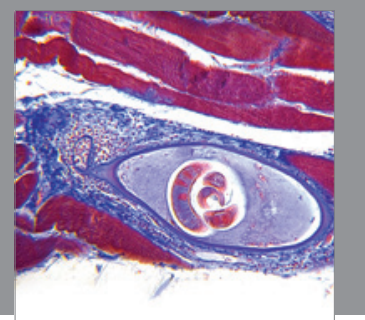

Gastroenterology

Research and Practice
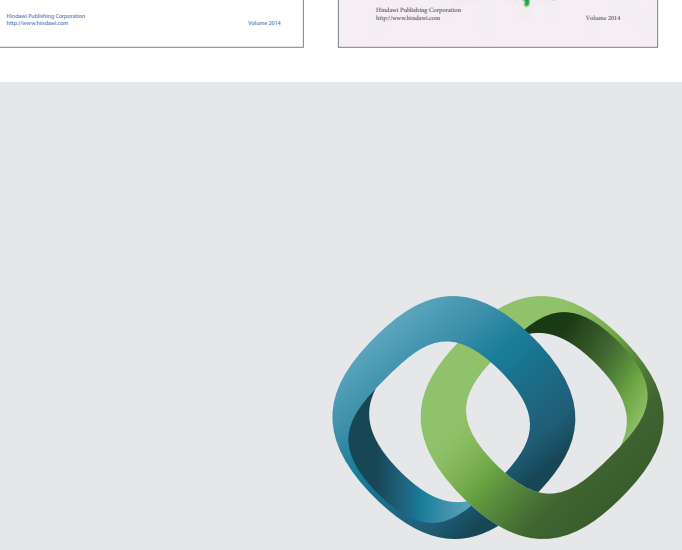

\section{Hindawi}

Submit your manuscripts at

http://www.hindawi.com
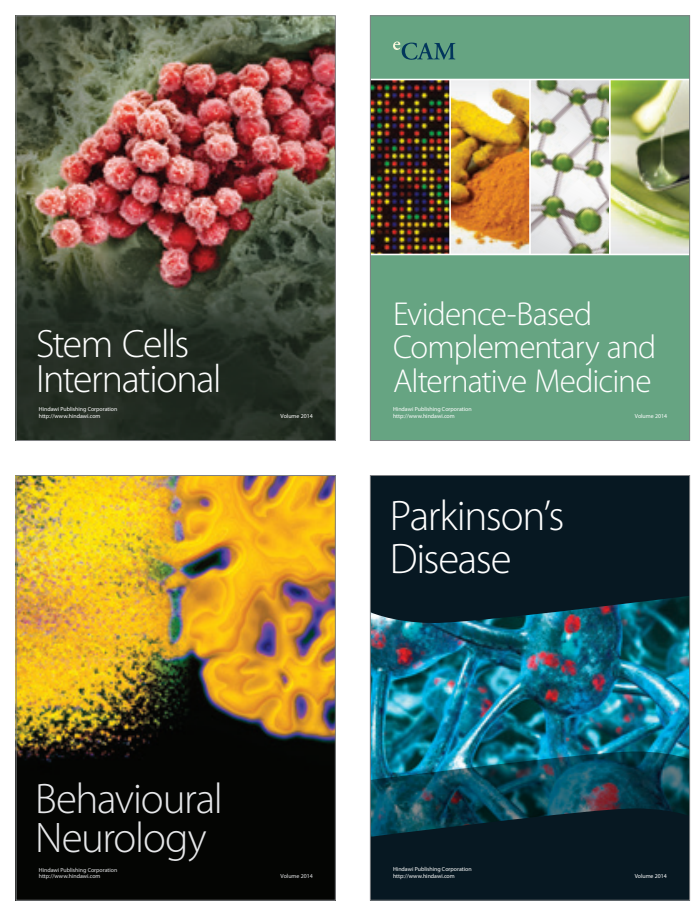

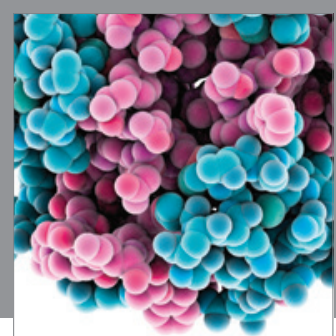

Journal of
Diabetes Research

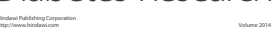

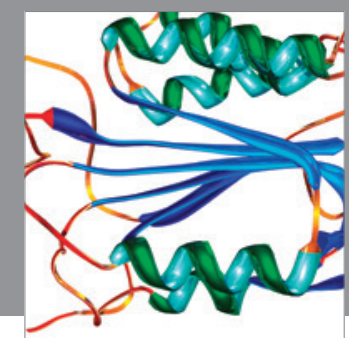

Disease Markers
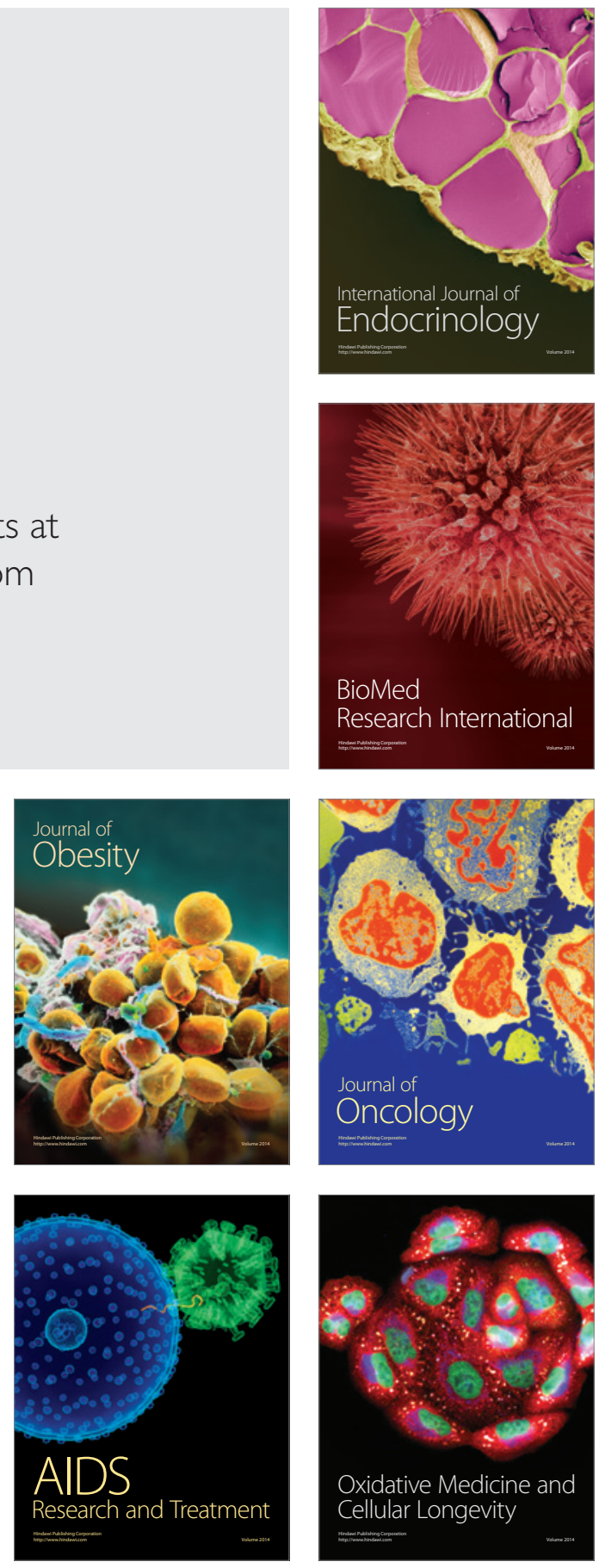